\title{
Evolução temporal da cobertura florestal na bacia hidrográfica do rio Iconha, Espírito Santo, Brasil
}

Felício Santos de Oliveira ${ }^{1}$

Ewerthon Mattos Paterlini²

Helena Furtado Pessoa de Mendonça ${ }^{3}$

Renan Pereira Barbosa 4

Alexandre Rosa dos Santos 5

\section{Resumo}

O presente trabalho objetiva avaliar a evolução temporal da cobertura florestal da bacia hidrográfica do rio Iconha, localizado no estado do Espírito Santo, Brasil, entre os anos de 1986 e 2010, utilizando geotecnologias. Para obtenção da evolução temporal, foram realizadas a obtenção e a correção das imagens do sensor LANDSAT 5, a fotointerpretação, a classificação dos fragmentos florestais e a elaboração do modelo digital de elevação, utilizando o sensor ASTER. Foi verificado que a cobertura vegetal representava $14,16 \%$ da área total em 1986 e passou para 30,48\% em 2010, gerando um incremento de $16,32 \%$ de cobertura florestal na área total. O município de Rio Novo do Sul, dentre os municípios abrangidos pela bacia, apresentou o maior percentual de recuperação, com 22,57\%. Áreas com altitudes entre 251 e 500 metros se mostraram mais significativas para a evolução da cobertura vegetal, representando $41 \%$ do incremento.

Palavras-chave: Dinâmica da paisagem. Sensoriamento remoto. Análise ambiental.

\section{Introdução}

De acordo com Santos (2007), toda paisagem possui uma dada configuração espacial e temporal em função do arranjo e das inter-relações entre os seus componentes. Se um ou mais desses componentes são modificados, a configuração espacial também será alterada e uma nova estrutura de paisagem será posta. Pode-se extrair de uma paisagem uma grande quantidade de informações, considerando todos os seus aspectos e a complexidade que a envolve. Em estudos de paisagem, pode-se analisar, por exemplo, sua geologia, os tipos de solo e de relevo, o sistema hídrico, além de algumas características climáticas.

0 monitoramento da modificação da paisagem torna-se uma ferramenta de análise para o entendimento da sua evolução, bem como a previsão de tendências futuras (SANTOS et al., 1981). A fragmentação florestal exerce diversos efeitos sobre a biota, altera a diversidade e a composição das comunidades nos fragmentos, influencia os processos ecológicos, como a polinização, a ciclagem de nutrientes e o estoque de carbono. (LAURANCE; VASCONCELOS, 2009).

\footnotetext{
$1 \quad$ Universidade Federal do Espírito Santo, mestrando em Ciências Florestais. Jerônimo Monteiro, Espírito Santo, Brasil. felicioliveira@ gmail.com. (28) 98100-1226. Av. Governador Lindemberg, 316, CEP: 29550-000, Jerônimo Monteiro, ES.

2 Universidade Federal do Espírito Santo, mestrando em Ciências Florestais. Jerônimo Monteiro, Espírito Santo, Brasil. ewerthon. eim@gmail.com. (28) 98118-3618. Av. Governador Lindemberg, 316, CEP: 29550-000, Jerônimo Monteiro, ES.

3 Universidade Federal do Espírito Santo,mestranda em Ciências Florestais. Jerônimo Monteiro, Espírito Santo, Brasil. helenafurtpessoa@gmail.com. (31) 3227-7997. Rua Turibaté, 213/301, Sion, CEP: 30315-410, Belo Horizonte, MG.

$4 \quad$ Universidade Federal do Espírito Santo, mestrando em Ciências Florestais. Jerônimo Monteiro, Espírito Santo, Brasil. renanpb_mg@ hotmail.com (28) 3558-2528. Av. Governador Lindemberg, 316, CEP: 29550-000, Jerônimo Monteiro, ES.

5 Universidade Federal do Espírito Santo, docente do Departamento de Engenharia Rural da UFES, Centro de Ciências Agrárias, Alegre, Espírito Santo, Brasil. mundogeomatica@yahoo.com.br (28) 3558-2528. Av. Governador Lindemberg, 316, CEP: 29550-000, Jerônimo Monteiro, ES.
} 
A ideia de integrar sensoriamento remoto e SIG (Sistemas de Informações Geográficas), para estruturar e manipular bases de dados, tem auxiliado na tarefa de mapeamento de habitats de várias espécies de fauna do Brasil. Segundo Crosta (1992), a utilização de imagens de sensoriamento remoto no monitoramento ambiental em escalas locais, regionais e globais é viável, principalmente pela rapidez, eficiência, periodicidade e visão sintética.

A partir de 1810, a região que compreende hoje o município de Iconha, que até então era coberta por Mata Atlântica e habitada por indígenas, começa a sofrer a ação de colonizadores, principalmente portugueses. A colonização do território de Iconha iniciou-se do litoral para o interior, estabelecendo-se ao longo dos rios e à proporção que as terras eram cultivadas, a região se desenvolvia. Foi essa penetração interiorana que deu origem ao povoado de Iconha, provavelmente em meados do século XIX. Seguindo esse processo, a região foi explorada, especialmente por imigrantes, dando início à cultura do café (CAPRINI, 2007).

Diante do exposto, este trabalho objetiva avaliar a evolução temporal - entre os anos de 1986 e 2010 - da cobertura florestal da bacia hidrográfica do rio Iconha, localizado no estado do Espírito Santo, Brasil, utilizando geotecnologias, com o propósito de indicar qual o histórico da fragmentação florestal, em um período de 24 anos, para constatação do acréscimo ou decréscimo da cobertura florestal dos municípios da região, bem como para a avaliação da presença desses fragmentos por classes de altitude.

\section{Material e métodos}

\section{Caracterização da área de estudo}

A bacia hidrográfica do rio Iconha possui uma área de $208 \mathrm{~km}^{2}$ e comprimento aproximado de $24 \mathrm{~km}$ e está inteiramente inserida no bioma Mata Atlântica. Localiza-se entre os municípios de Iconha, Rio Novo do

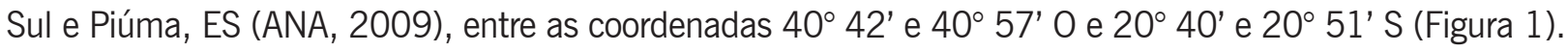
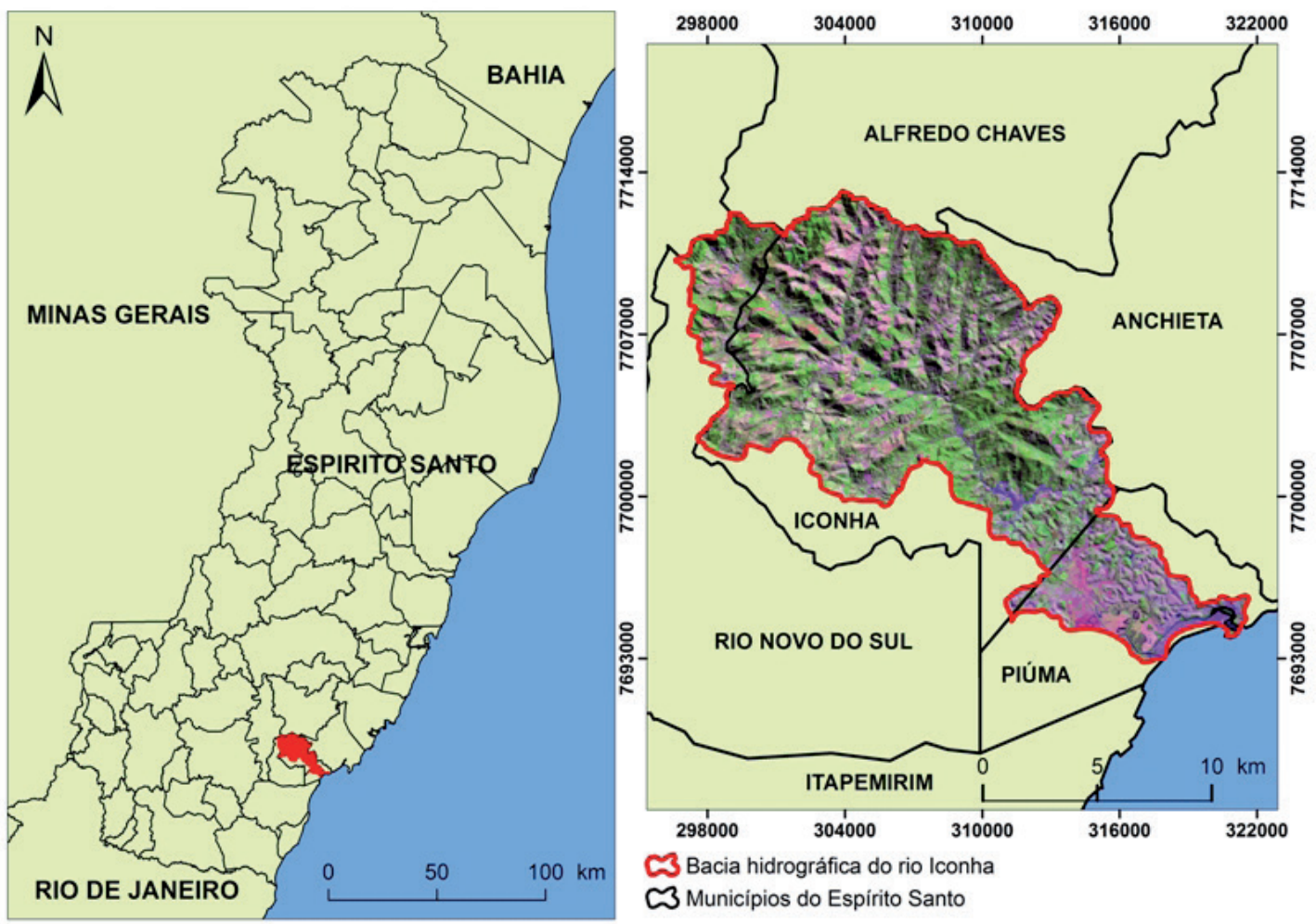

Figura 1. Localização da bacia hidrográfica do rio Iconha, ES.

Fonte: Elaboração dos autores. 
Aproximadamente $77 \%$ da bacia hidrográfica do rio Iconha encontra-se no município de Iconha, região Sul do Estado do Espírito Santo. Esse município limita-se ao norte com os municípios de Alfredo Chaves e Anchieta, ao sul com os municípios de Rio Novo do Sul e Piúma e a oeste com Rio Novo do Sul. O rio Iconha é um afluente do Rio Novo, possui a foz no município de Piúma e é também transpassado pela BR 101 (CAPRINI, 2007).

\section{Obtenção e correção das imagens}

Para inicialização da análise e interpretação da evolução multitemporal da fragmentação florestal na bacia do rio Iconha, foi feita uma seleção de imagens do sensor LANDSAT 5 - Thematic Mapper, bandas 3, 4 e 5. Essas imagens foram obtidas por meio do site da Agência Espacial Americana (National Aeronautic and Space Administration - NASA) em parceria com o Serviço Geológico Americano (United States Geological Survey - USGS). Foram utilizadas duas épocas de passagem do satélite, referentes aos dias 05/11/1986 e 31/05/2010, totalizando um intervalo de 24 anos. Vale ressaltar que as datas das duas passagens são diferentes (novembro e maio), o que sugere alterações nos ângulos de energia incidentes sobre os alvos analisados nas imagens.

As imagens capturadas pelo sensor sofrem influência de diversos fatores na atmosfera, tais como poeiras, partículas suspensas e aerossóis, o que ocasiona a presença de pixels espúrios. Com o intuito de minimizar tais adversidades, realizou-se a correção atmosférica pela técnica de subtração de pixels escuros ou Dark Object Subctration (DOS) proposto por Chavez (1988).

Após a correção atmosférica das imagens, foi realizada a conversão dos valores dos níveis digitais presentes nas imagens para radiância. Posteriormente, as imagens com valores de radiância foram transformadas em imagens reflectância, tornando possível a avaliação da evolução da cobertura florestal entre os anos de 1986 e 2010. Essa etapa de transformação foi realizada por meio da aplicação da equação 1 (PRICE, 1987):

$$
\rho=\frac{\left(\Pi-L \lambda-d^{2}\right)}{(E S o l ~ \lambda-\cos \theta 0)}
$$

Em que,

$\rho$ é a reflectância medida ao nível do satélite, adimensional;

$\mathrm{L} \lambda$ é a radiância espectral no detetor, em w. $\mathrm{m}^{2} \mathrm{sr} . \mu \mathrm{m}$;

d é a distância Terra-Sol, em unidades astronômicas;

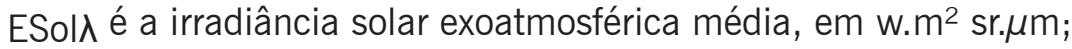

Өs é o ângulo zenital solar, em graus.

Os dados espaciais utilizados, como os limites municipais, a bacia hidrográfica e os cursos d'água, foram obtidos pelo GEOBASES, que inclui as curvas de nível de $50 \mathrm{~m}$ em $50 \mathrm{~m}$ e a rede hidrográfica. A ortofoto, utilizada para auxílio na tomada de decisões durante o processo de fotointerpretação dos fragmentos florestais, foi disponibilizada pelo IEMA (2009).

\section{Fotointerpretação e classificação dos fragmentos florestais}

Para o processo de reconhecimento de fragmentos florestais na imagem, utilizou-se uma classificação supervisionada por meio do software ArcMap 10.1. A classificação supervisionada consiste 
no estabelecimento de um processo de decisão no qual um grupo de pixels é definido como uma determinada classe (PEREIRA et al., 2013). Utilizando-se de algoritmos, o programa computacional associa os grupos de pixels semelhantes à sua determinada classe, definida anteriormente por meio de um conjunto de amostras de treinamento.

Adiante, seguem os passos para a realização da classificação supervisionada de fragmentos florestais:

- localização de exemplos representativos de fragmentos florestais identificados na imagem;

- digitalização em tela dos polígonos em torno de cada célula de treinamento;

- definição de uma assinatura espectral para os fragmentos florestais;

- classificação de toda a imagem, considerando cada pixel individualmente, e, assim, comparando sua assinatura particular com as assinaturas conhecidas.

Para realização da última etapa, foi utilizado o algoritmo da máxima verossimilhança com o intuito de classificar as imagens orbitais dos anos estudados. Por meio desse algoritmo, foi possível calcular a probabilidade estatística de um dado valor de pixel pertencer a uma classe particular de cobertura da terra, encontrando, dessa forma, todas as áreas de fragmentos florestais presentes nas imagens dos anos de 1986 e 2010.

Com a classificação supervisionada concluída, foram gerados mapas vetoriais poligonais representativos da fragmentação florestal para os anos de 1986 e 2010, onde foi quantificada a área dos fragmentos florestais na bacia do rio Iconha entre o intervalo dos 24 anos avaliados.

\section{Modelo digital de elevação}

O Modelo Digital de Elevação (MDE) da bacia hidrográfica do rio Iconha, adotado para este estudo, foi elaborado a partir de dados do sensor ASTER (Advanced Space Borne Thermal Emission and Reflection Radiometer), a bordo do satélite Terra. Esses modelos digitais de elevação são oriundos de pares estereoscópicos de imagens do ASTER, sensor VNIR (dados na faixa do infravermelho próximo). Apresenta resolução espacial de 30 metros, sem a necessidade de interpolação. As imagens ASTER são oriundas de imagens orbitais e, dessa forma, o ASTER GDEM está vulnerável às interferências ocasionadas por nuvem (VALERIANO, 2004).

De posse do MDE, foram delimitadas cinco classes de altitudes para avaliação da mudança da cobertura vegetal por gradiente de elevação, sendo elas: menores que 100m, 101 a 250m, 251 a $500 \mathrm{~m}, 501$ a $1.000 \mathrm{~m}$ e maiores que $1.000 \mathrm{~m}$.

\section{Resultados e discussão}

A Figura 2 representa a evolução espaço-temporal dos fragmentos florestais da bacia hidrográfica do rio Iconha. O mapa destaca as áreas onde ocorreram as modificações, sem uma contribuição detalhada sobre o tipo de alteração ou técnica utilizada para tal.

Ao comparar os dois períodos, constatou-se que a cobertura florestal na área de estudo aumentou. A área de cobertura florestal, que em 1986 representava $14,16 \%$ da área total, passou para $30,48 \%$, representando um aumento de 16,32\%. Esse aumento decorre principalmente da substituição das áreas de capoeira e pastagem por fragmentos florestais. 


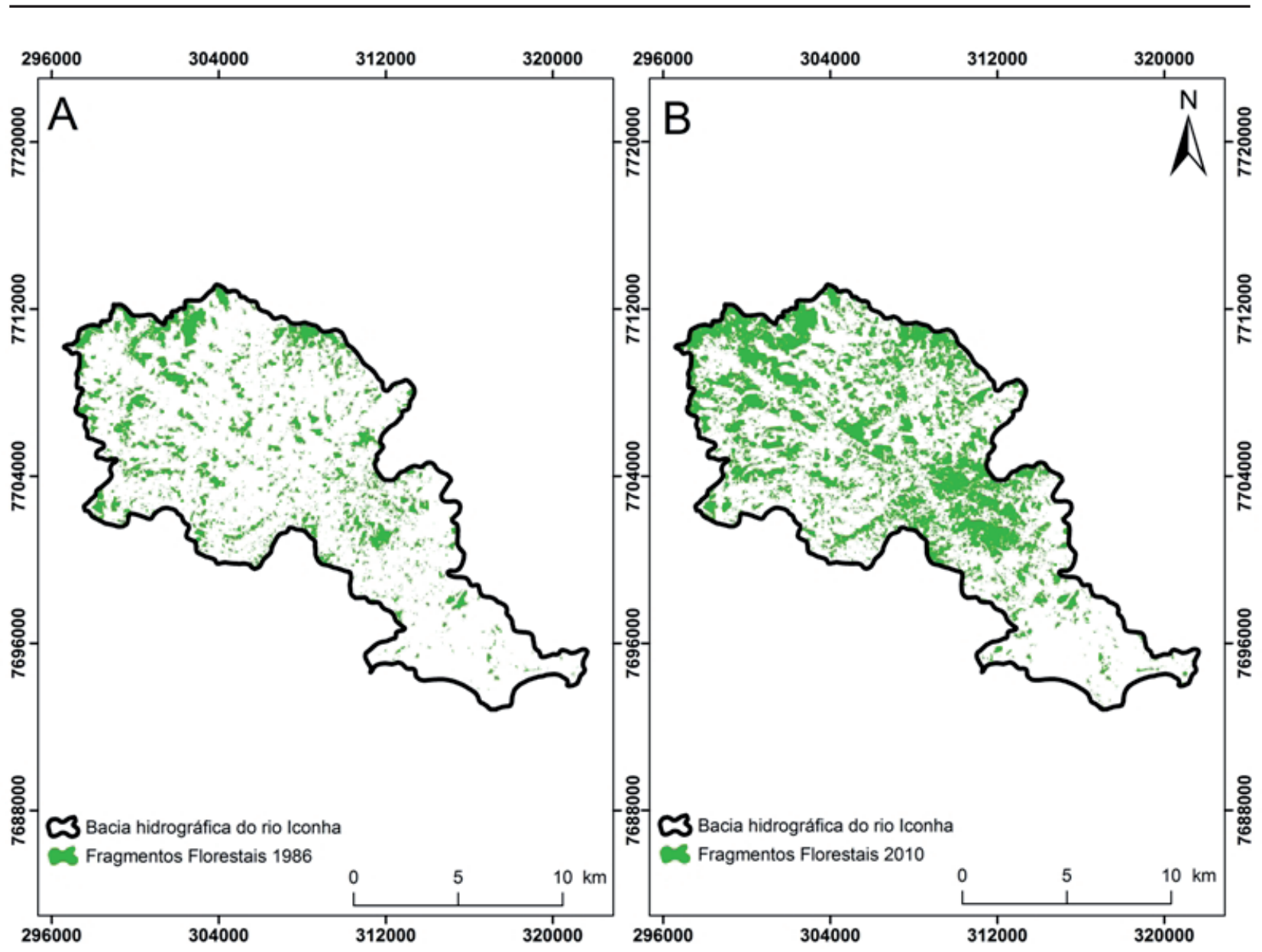

Figura 2. Fragmentos Florestais da bacia hidrográfica do rio Iconha, nos anos 1986 (A) e 2010 (B). Fonte: Elaboração dos autores.

Visando obter os principais colaboradores para o incremento da cobertura florestal da região, foram calculadas as porcentagens de acréscimo da vegetação por municípios pertencentes à bacia hidrográfica. A Tabela 1 representa o incremento dos fragmentos florestais por área dos três municípios que compõem a bacia hidrográfica do rio Iconha.

Tabela 1. Evolução dos fragmentos florestais por municípios

\begin{tabular}{llll}
\hline \multirow{2}{*}{ Municípios } & \multicolumn{3}{l}{ Fragmentos Florestais (\%) } \\
\cline { 2 - 4 } & $\mathbf{1 9 8 6}$ & $\mathbf{2 0 1 0}$ & Total \\
\hline Iconha & 15,18 & 33,33 & 18,15 \\
Piúma & 3,14 & 6,1 & 2,96 \\
Rio Novo do Sul & 23,55 & 46,12 & 22,57 \\
\hline
\end{tabular}

Fonte: Elaboração dos autores.

O aumento da cobertura de vegetação pode ser explicado pelo aumento da área de plantios florestais, como o de eucalipto, pois a assinatura espectral é semelhante à da vegetação nativa, também encontrada no município de Santa Maria de Jetibá, no Espírito Santo (SANTOS et al., 2012).

Coura (2006) expõe que a confusão espectral de certas classes de floresta pode levar a uma diminuição da exatidão do mapeamento das feições e, embora haja semelhança espectral entre 
plantios florestais e fragmentos de florestas nativas, há características que os distinguem. Plantios florestais apresentam cobertura mais homogênea e distribuição e formas regulares, indo de encontro com a irregularidade da vegetação nativa.

De posse das análises realizadas na Figura 3, por meio de ferramentas de calculadora de mapas, podemos observar que a bacia hidrográfica do rio Iconha tem o ponto mais elevado a 1.031 metros de altitude, mas apenas $0,01 \%$ da área possui altitudes acima de $1.000 \mathrm{~m}$, constituindo, assim, a menor área identificada. A classe de altitudes médias 251 a 500 metros representa 30,82\% da área, e a classe 501 a 1.000 metros, apresenta 33,74\% da área total, estabelecendo-se então como a maior área encontrada.
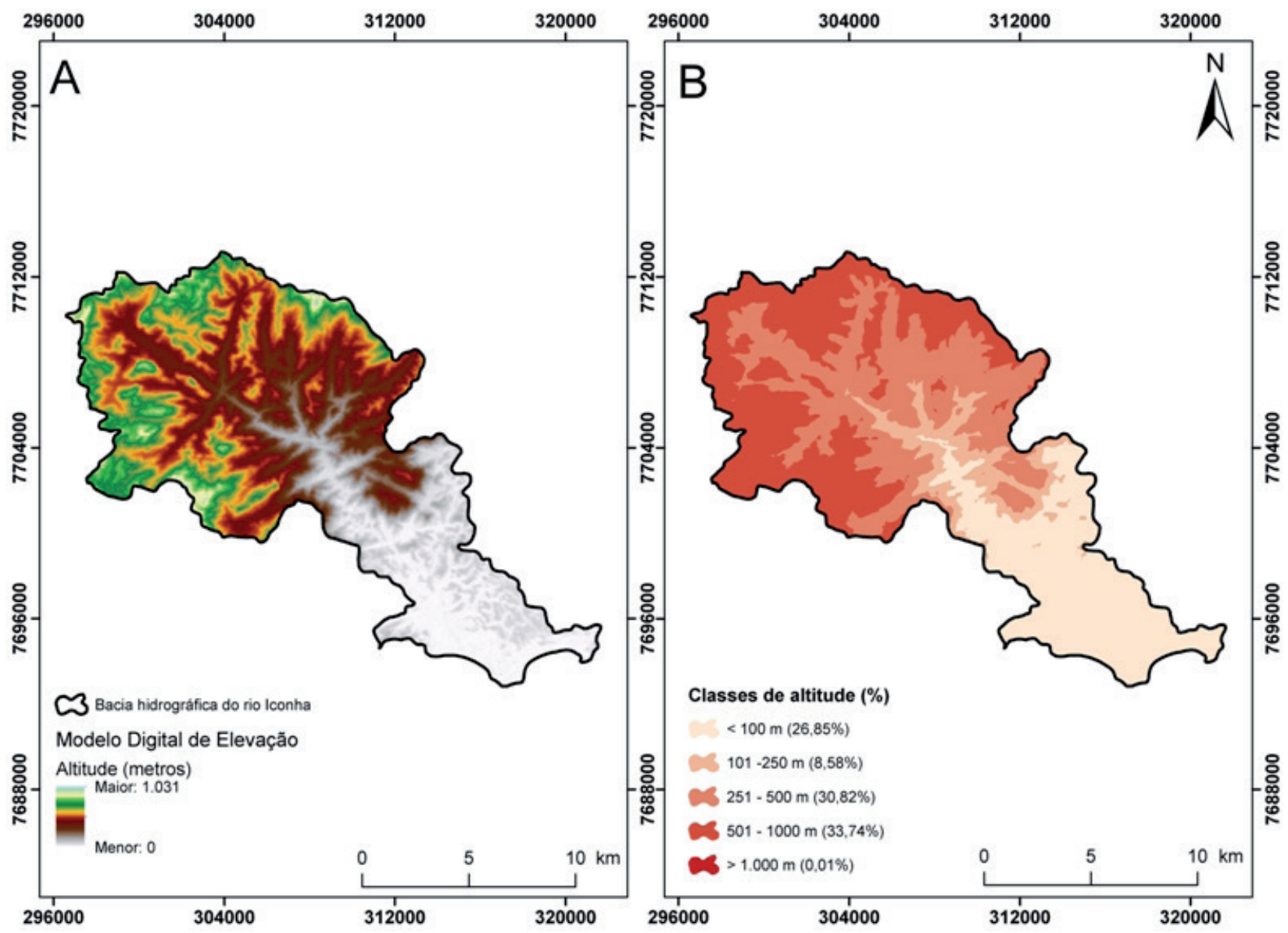

Figura 3. Modelo digital de elevação $(A)$ e classes de altitude $(B)$ da bacia hidrográfica do rio Iconha. Fonte: Elaboração dos autores. 


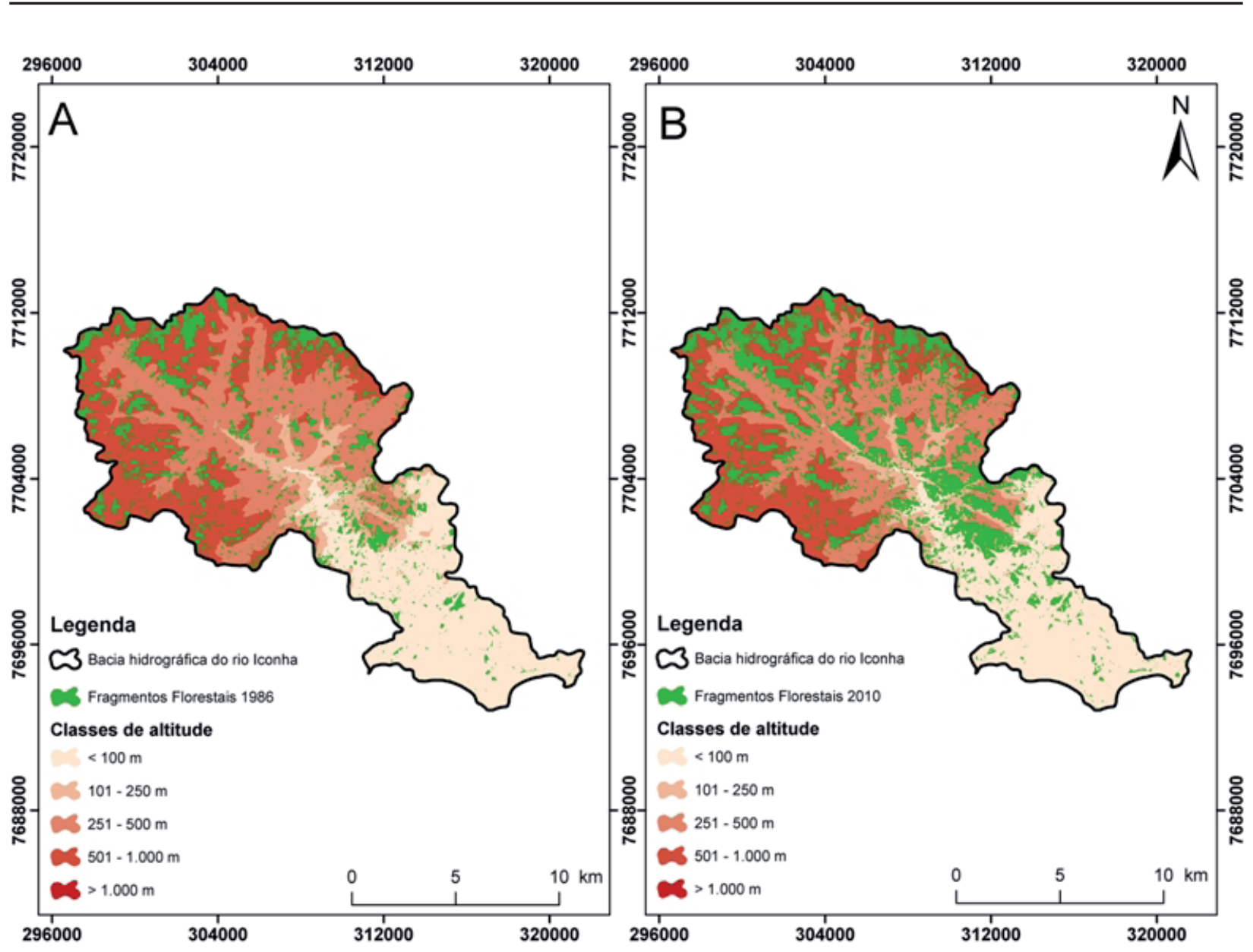

Figura 4. Fragmentos florestais por classes de altitude da bacia hidrográfica do rio Iconha, nos anos 1986 (A) e 2010 (B).

Fonte: Elaboração dos autores.

A Tabela 2 apresenta o incremento dos fragmentos florestais, em hectares, por classes de altitude da bacia hidrográfica do rio Iconha.

Tabela 2. Evolução dos fragmentos florestais por gradiente de altitude

\begin{tabular}{|c|c|c|c|}
\hline \multirow{2}{*}{ Classes de Altitude (m) } & \multicolumn{3}{|c|}{ Fragmentos Florestais (ha) } \\
\hline & 1986 & 2010 & Acréscimo \\
\hline$<100 \mathrm{~m}$ & 251,72 & 584,50 & 332,78 \\
\hline $101-250 m$ & 247,40 & 719,50 & 472,10 \\
\hline $251-500 m$ & 692,29 & 1631,94 & 939,65 \\
\hline $501-1.000 \mathrm{~m}$ & 1754,66 & 2301,64 & 546,97 \\
\hline$>1.000 \mathrm{~m}$ & 0,09 & 0,18 & 0,09 \\
\hline
\end{tabular}

Fonte: Elaboração dos autores.

Este resultado tornou-se positivo no aspecto da evolução da cobertura vegetal, uma vez que, em alguns estudos, como o realizado na bacia do rio Itajaí (VIBRANS, 2003), o desmatamento de florestas e a exploração seletiva foram realizadas na classe de altitudes médias, entre 300 e $600 \mathrm{~m}$. 


\title{
Conclusões
}

Por meio deste trabalho, verificou-se que as áreas de capoeira e pastagem foram substituídas, na passagem temporal estudada, por fragmentos florestais, o que pode ser verificado pelo incremento apresentado.

Em comparação com outros estudos, foi possível verificar que, nas regiões mais comumente expostas ao desmatamento de florestas e à exploração seletiva, houve o maior percentual no acréscimo dos fragmentos florestais, o que auxilia na manutenção da biodiversidade da bacia hidrográfica.

\section{Temporal evolution of Iconha river forest cover, Espírito Santo, Brazil}

\begin{abstract}
This paper aims to evaluate the temporal evolution of the forest cover in Iconha river catchment, located in Espírito Santo state, Brazil, between 1986 and 2010, using geotechnology. In order to get the time evolution, we obtained and corrected the Landsat 5 sensor images, we also analyzed the photos and classified forest fragments and the development of digital elevation model using ASTER sensor. It was found that vegetation represented $14.16 \%$ of the total area in 1986 and rose to $30.48 \%$ in 2010 , resulting in an increase of $16.32 \%$ forest cover in total area. Among all the counties covered by the basin, Rio Novo do Sul had the highest percentage of recovery $(22.57 \%)$. Areas with altitudes between 251 and 500 meters were more significant for the evolution of vegetation, representing $41 \%$ of the increase.
\end{abstract}

Key words: Landscape dynamics. Remote sensing. Environmental analysis.

\section{Referências}

ANA - AGÊNCIA NACIONAL DE ÁGUAS - ANA. Programa Nacional de Avaliação da Qualidade das Águas. 2009. Disponível em: <http://mapas.ana.gov.br/pnqa/>. Acesso em: 27 jun. 2013.

CHAVEZ, J. P. S. An improved dark-object subtraction technique for atmospheric scattering correction of multiescptral data. Remote Sensing of Environment. v. 24, p. 454-479, 1988.

COURA, S. M. C. Mapeamento de vegetação do estado de Minas Gerais utilizando dados MODIS. 2006. 129 f. 2006. Dissertação (Mestrado em Sensoriamento remotoRemoto) - Instituto Nacional de Pesquisas Espaciais. , São José dos Campos: INPE, 2006..

CRÓSTA, A. P. Processamento digital de imagens de sensoriamento remoto. Campinas, SP: IG/UNICAMP, 1992. $170 \mathrm{p}$.

CAPRINI, A. B. A. O Comércio como propulsor do poder político em Iconha: o coronel Antônio Duarte (1889-1915). 2007. 139 f. Dissertação (Mestrado em História) - Centro de Ciências Humanas e Naturais, Universidade Federal do Espírito Santo, Vitória, ES.

IEMA Ortofotomosaico 2007/2008, 2009. Convênio "Vale de qualidade ambiental". Companhia Vale e Instituto Estadual de Meio Ambiente e Recursos Hídricos-IEMA. 
LAURANCE, W. F.; VASCONCELOS, H. L. Consequências Ecológicas da Fragmentação Florestal na Amazônia. Oecologia Brasiliensis. Rio de Janeiro, RJ. v. 3, n. 4, p. 434-451, 2009.

NATIONAL AERONAUTICS AND SPACE ADMINISTRATION - NASA. 2013. Disponível em: < https:// zulu.ssc.nasa.gov/mrsid/mrsid.pl>. Acesso em: 15 jun. 2013.

PRICE, J. C. Radiometric calibration of satellite sensors in the visible and near infrared: history and outlook. Remote Sensing of Environment, v. 22, p. 3-9, 1987.

PEREIRA, R. A.; ALCÂNTARA, C. R.; NETO, J. D.; BARBOSA, E. M. Análise espaço-temporal da cobertura vegetal e do avanço de Prosopisjuliflora (SW) DC numa área de caatinga. Ra'e Ga - 0 Espaço Geográfico em Análise. v 28. p 154-180. 2013.

SANTOS, R. F. (Org.). Vulnerabilidade Ambiental- Desastres Naturais ou Fenômenos Induzidos? Brasília: MMA, 2007. 192p

SANTOS, A. R.; ALMEIDA, H. C. J.; EUGÊNIO, F. C. Evolução da Cobertura Florestal no Município de Santa Maria de Jetibá - ES. Floram - Floresta e Ambiente, Seropédica, RJ, v. 19, n. 3, p. 296-307, 2012.

SANTOS, A. P.; NOVO, E. M.; LOMBARDO, M. A. Metodologia de Interpretação de Dados de Sensoriamento Remoto e Aplicações no Uso da terra. In: SIMPÓSIO BRASILEIRO DE SENSORIAMENTO REMOTO. 7, 1981, São José dos Campos. Anais... São José dos Campos, SP. 1981, p. 172-175.

VALERIANO, M. M. Modelo digital de elevação com dados SRTM disponíveis para a América do Sul. São José dos Campos, SP. INPE: Coordenação de Ensino, Documentação e Programas Especiais, 2004. p. 72. (INPE-10550-RPQ/756).

VIBRANS, A. C. A cobertura florestal da bacia hidrográfica do rio Itajaí - elementos para uma análise histórica. 2003. 239 f. Tese de doutorado (Doutorado em Geografia). Universidade Federal de Santa Catarina, Centro de Filosofia e Ciências Humanas, Florianópolis, SC.

UNITED STATES GEOLOGICAL SURVEY - USGS. 2013. Disponível em: < http://earthexplorer.usgs. gov/>. Acesso em: 15 jun. 2013.

\section{Histórico editorial}

Submetido em: 22/09/2014

Aceito em: 22/01/2015 